\title{
Asymptotic Behavior of the Solutions of an Elliptic-Parabolic System Arising in Flow in Porous Media
}

\author{
Y. Amirat and A. Ziani
}

\begin{abstract}
We study the asymptotic behavior, with respect to high Péclet numbers, of the solutions of the nonlinear elliptic-parabolic system governing the displacement of one incompressible fluid by another, completely miscible with the first, in a porous medium. Using compensated compactness techniques, we obtain the existence of a global weak solution to the nonlinear degenerate elliptic-parabolic system modelling the flow when the molecular diffusion effects are neglected.
\end{abstract}

Keywords: Nonlinear degenerate elliptic-parabolic systems, miscible flow in porous media

MSC 2000: Primary 35D05, secondary 35K60, 35K65,76S05

\section{Introduction and formulation of the problem}

This paper is concerned with the mathematical analysis of a model describing fluid flow and transport processes in porous media that arise in petroleum engineering simulation or in subsurface contaminant transport. We consider a model problem consisting of the isothermal miscible displacement of an incompressible binary mixture.

Let $\Omega$ be a bounded domain of $\mathbb{R}^{d}(d=2,3)$ representing the porous reservoir, $(0, T)$ a time interval and $\Omega_{T}=\Omega \times(0, T)$. Under appropriate physical assumptions, the equations describing the displacement of one incompressible

Y. Amirat: Univ. Blaise Pascal, Lab. de Math. Appl., CNRS UMR 6620, F-63177 Aubière cedex, France; amirat@math.univ-bpclermont.fr

A. Ziani: Univ. de Nantes, Dépt. de Math., BP 92208, F-44322 Nantes cedex 3, France; ziani@math.univ-nantes.fr

ISSN 0232-2064 / \$2.50 C Heldermann Verlag Berlin 
fluid by another, completely miscible with the first, are given by

$$
\left.\begin{array}{rl}
\operatorname{div} u & =q^{+}-q^{-} \\
u & =-\frac{\kappa(x)}{\mu(c)} \operatorname{grad} p \\
\operatorname{grad} c) & =\hat{c} q^{+}
\end{array}\right\}
$$

for $(x, t) \in \Omega_{T}$. We refer to Bear [3], Chavent and Jaffré [4], Douglas [7], and Scheidegger [16] for a detailed description of the model.

Here, the gravitational terms are omitted for simplicity of exposition, $p$ is the pressure in the fluid mixture, $u$ is the Darcy velocity, $c$ denotes the concentration of one of the two components of the fluid mixture, $\kappa=\kappa(x)$ is the absolute permeability of the rock, $\phi=\phi(x)$ is the porosity, and $\mu$ is the concentration-dependent viscosity. $\phi$ and $\kappa$ (case of isotropy) are arbitrary bounded measurable functions on $\Omega$ that satisfy

$$
\left.\begin{array}{l}
0<\phi_{*} \leq \phi(x) \leq \phi_{*}^{-1} \\
0<\kappa_{*} \leq \kappa(x) \leq k_{*}^{-1}
\end{array}\right\} \quad(x \in \Omega)
$$

where $\phi_{*}$ and $\kappa_{*}$ are given real numbers. Note that, by definition,

$$
0 \leq c(x, t) \leq 1 \quad\left((x, t) \in \Omega_{T}\right)
$$

The viscosity $\mu=\mu(c)$ is assumed to follow the quarter-power mixing rule, widely used (see Koval [12])

$$
\mu(c)=\mu(0)\left(1+\left(M^{\frac{1}{4}}-1\right) c\right)^{-4} \quad(c \in(0,1))
$$

where $M=\frac{\mu(0)}{\mu(1)}$ is the mobility ratio. The stability of the flow is characterized by the mobility ratio; it has been observed experimentally that for $M>1$ instabilities in the flow will grow into viscous fingers.

The functions $q^{+}$and $q^{-}$are the injection and production source terms, respectively, and $\hat{c}$ is specified at the sources and is equal to the resident concentration at the sinks.

The hydrodynamic dispersion tensor $D(x, u)$ has the form

$$
\begin{aligned}
D(x, u) & =\phi(x)\left(d_{m} I+D^{\circ}(u)\right) \\
D^{\circ}(u) & =|u|\left(d_{l} E(u)+d_{t}(I-E(u))\right)
\end{aligned}
$$

where $I$ is the identity matrix, $E$ is the matrix $\left(\frac{u_{i} u_{j}}{|u|^{2}}\right), d_{m}$ is the molecular diffusion coefficient, $D^{\circ}(u)$ is the mechanical dispersion tensor, $d_{l}$ and $d_{t}\left(d_{l} \geq\right.$ 
$d_{t}$ ) are the longitudinal and transverse dispersion coefficients, respectively. We notice that the hydrodynamic dispersion tensor satisfies

$$
\begin{aligned}
D(x, u) \xi \cdot \xi & \geq \phi_{*}\left(d_{m}+d_{t}|u|\right)|\xi|^{2} m \\
|D(x, u) \xi| & \leq \phi_{*}^{-1}\left(d_{m}+d_{l}|u|\right)|\xi|
\end{aligned}
$$

for $\xi \in \mathbb{R}^{d}$ and $x \in \Omega$. At relatively high flow velocities, corresponding to Péclet numbers $P e \gg 1$, the effects of mechanical dispersion are much greater than those of molecular diffusion, the contribution of molecular diffusion often is negligible (see Bear [3], Anderson [2], Coutinho and Alvez [6], Pearson and Tardy [15], Shubin and Bell [17], and Young [19]).

Equation $(1.1)_{1}$ (with $(1.1)_{2}$ ) is the pressure equation derived from the conservation of the total mass and $(1.1)_{3}$ is the concentration equation derived from the conservation of mass for one of the two components of the mixture. The pressure equation is of elliptic nature while the concentration equation is an advection-diffusion equation, advection being the dominant phenomenon.

System (1.1) is provided with the boundary and initial conditions

$$
\left.\begin{array}{rlrl}
u \cdot \nu & =0 & \\
D(x, u) \operatorname{grad} c \cdot \nu & =0 & & \text { on } \Gamma_{T} \\
c(x, 0) & =c_{0}(x) & \text { on } \Omega
\end{array}\right\}
$$

where $\Gamma_{T}=\Gamma \times(0, T), \Gamma$ denoting the boundary of $\Omega$, and $\nu$ is the unit normal pointing outward $\Omega$. Since the pressure is only determined up to a constant we additionally require that the pressure is normalized, i.e. $\int_{\Omega} p(x, t) d x=0$ for all $t \in(0, T)$.

The aim of the present paper is to report on the case where the molecular diffusion is neglected. One important character of the corresponding mathematical model is the possible degeneracy, in the stagnant zone, of the second order diffusion operator in the concentration equation. The diffusion operator may be zero pointwise, it can be small or zero in regions of the solution space, and fairly large for other values of the solution. Consequently, solutions of the concentration equation will, in general, possess minimal smoothness. The main difficulty in studying system (1.1) is due to the strong coupling of the equations, we do not know a priori the regions where $u$ has zero, small or large values.

We make the following assumptions:

(A1) $\Omega$ is a simply connected bounded domain in $\mathbb{R}^{d}(d \leq 3)$ with boundary $\Gamma$ in the class $C^{1,1}$.

(A2) $c_{0} \in L^{\infty}(\Omega)$ and $0 \leq c_{0}(x) \leq 1$ a.e. in $\Omega$. 
(A3) $\hat{c} \in L^{\infty}\left(\Omega_{T}\right)$ and $0 \leq \hat{c}(x, t) \leq 1$ a.e. in $\Omega_{T}$.

(A4) $q^{+}, q^{-} \in L^{\infty}\left(0, T ; L^{2}(\Omega)\right)$ and $\int_{\Omega}\left(q^{+}-q^{-}\right) d x=0$.

(A5) The function $\mu \in C^{2}([0,1])$ is such that $\mu$ and $\frac{1}{\mu}$ are strictly convex and $0<\mu_{-} \leq \mu(c) \leq \mu^{+}$for all $c \in(0,1)$.

where $\mu_{-}$and $\mu^{+}$are two fixed real numbers. Obviously, the function $\mu$ defined by (1.3) satisfies (A5). In addition, we suppose the porosity and the permeability of the medium constant and equal to 1 ; the same analysis applies at least for $\kappa$ in $W^{1, \infty}(\Omega)$ and $\phi$ satisfying (1.2).

We first recall the following well-known result (see Mikelić [14], Fabrie and Langlais [8], Feng [9], and Chen and Ewing [5]).

Theorem 1.1. Assume that hypotheses (A1) - (A5) hold, $d_{l} \geq d_{t}>0$ and $d_{m}>0$. Then there exists a pair $(p, c)$ of functions $p \in L^{\infty}\left(0, T ; H^{1}(\Omega)\right)$ and $c \in L^{\infty}\left(0, T ; L^{2}(\Omega)\right) \cap L^{2}\left(0, T ; H^{1}(\Omega)\right)$, satisfying

$$
\begin{aligned}
& \int_{\Omega} p(x, t) d x=0 \\
& 0 \leq c(x, t) \leq 1 \text { for a.e. } x \in \Omega
\end{aligned} \quad(t \in(0, T))
$$

solution to system (1.1) provided with conditions (1.6).

In the sequel, we take $d_{m}=\varepsilon$ with $0<\varepsilon \ll 1$ and $d_{l} \geq d_{t}>0$ in (1.4), thus

$$
D\left(u^{\varepsilon}\right)=\varepsilon I+D^{\circ}\left(u^{\varepsilon}\right) .
$$

We consider the elliptic-parabolic system

$$
\left.\begin{array}{rl}
\operatorname{div} u^{\varepsilon} & =q^{+}-q^{-} \\
u^{\varepsilon} & =-\frac{1}{\mu\left(c^{\varepsilon}\right)} \operatorname{grad} p^{\varepsilon} \\
\left.u^{\varepsilon} \cdot \nu\right|_{\Gamma_{T}} & =0 \\
\int_{\Omega} p^{\varepsilon} d x & =0 \\
\partial_{t} c^{\varepsilon}+u^{\varepsilon} \cdot \operatorname{grad} c^{\varepsilon}+c^{\varepsilon} q^{+}-\operatorname{div}\left(D\left(u^{\varepsilon}\right) \operatorname{grad} c^{\varepsilon}\right) & =\hat{c} q^{+} \\
\left.D\left(u^{\varepsilon}\right) \operatorname{grad} c^{\varepsilon} \cdot \nu\right|_{\Gamma_{T}} & =0 \\
\left.c^{\varepsilon}\right|_{t=0} & =c_{0}
\end{array}\right\}
$$

We examine the asymptotic behavior, as $\varepsilon \rightarrow, 0$, of the weak solutions $\left(p^{\varepsilon}, c^{\varepsilon}\right)$ of this problem. The main objective of the paper is to prove the existence of a weak solution, in the sense hereafter, to problem (1.7) in the case $\varepsilon=0$, that is when the molecular diffusion effects are neglected. The limit problem 
is a degenerate elliptic-parabolic system which writes as

$$
\left.\begin{array}{rl}
\operatorname{div} u & =q^{+}-q^{-} \\
u & =-\frac{1}{\mu(c)} \operatorname{grad} p \\
\left.u \cdot \nu\right|_{\Gamma_{T}} & =0 \\
\int_{\Omega} p d x & =0 \\
\partial_{t} c+u \cdot \operatorname{grad} c+c q^{+}-\operatorname{div}\left(D^{\circ}(u) \operatorname{grad} c\right) & =\hat{c} q^{+} \\
\left.D^{\circ}(u) \operatorname{grad} c \cdot \nu\right|_{\Gamma_{T}} & =0 \\
\left.c\right|_{t=0} & =c_{0}
\end{array}\right\}
$$

where $D^{\circ}(u)$ is the mechanical dispersion tensor which writes also

$$
D^{\circ}(u)=\left(d_{l}-d_{t}\right) \frac{u u^{\top}}{|u|}+d_{t}|u| I .
$$

Definition 1.2. A pair $(p, c)$ is said to be a weak solution of Problem (1.8) if:

(i) $p \in L^{\infty}\left(0, T ; H^{1}(\Omega)\right)$ and $p$ is a solution of the elliptic problem defined by the first four equations of system (1.8).

(ii) $c \in L^{\infty}\left(\Omega_{T}\right)$ with $0 \leq c(x, t) \leq 1$ a.e. on $\Omega_{T}$, with $|u|^{\frac{1}{2}} \operatorname{grad} c \in$ $\left(L^{2}\left(\Omega_{T}\right)\right)^{d}$, and $c$ satisfies the last three equations of system (1.8) in the sense that

$$
\begin{gathered}
\int_{\Omega_{T}}\left\{c \partial_{t} \varphi+c u \cdot \operatorname{grad} \varphi-D^{\circ}(u) \operatorname{grad} c \cdot \operatorname{grad} \varphi-c q^{-} \varphi\right\} d x d t \\
=-\int_{\Omega_{T}} \hat{c} q^{+} \varphi d x d t-\int_{\Omega} c_{0}(x) \varphi(x, 0) d x
\end{gathered}
$$

for any $\varphi \in C^{1}\left(\bar{\Omega}_{T}\right)$ with compact support in $\bar{\Omega} \times[0, T)$.

Note here that the conditions $u \in\left(L^{\infty}\left(0, T ; L^{2}(\Omega)\right)\right)^{d}$ and $|u|^{\frac{1}{2}} \operatorname{grad} c \in$ $\left(L^{2}\left(\Omega_{T}\right)\right)^{d}$ imply, by Hölder's inequality, that $|u| \operatorname{grad} c \in\left(L^{2}\left(0, T ; L^{\frac{4}{3}}(\Omega)\right)\right)^{d}$, and then, by the last inequality of (1.5) with $d_{m}=0$, that $D^{\circ}(u) \operatorname{grad} c \in$ $\left(L^{2}\left(0, T ; L^{\frac{4}{3}}(\Omega)\right)\right)^{d}$.

Our main result is the following one.

Theorem 1.3. Assume that hypotheses (A1) - (A5) hold. Let $\varepsilon>0$ and $d_{l} \geq d_{t}>0$ in (1.9) and let $\left(p^{\varepsilon}, c^{\varepsilon}\right)$ denote a corresponding weak solution to 
system (1.7). Then there are extracted subsequences from $\left(p^{\varepsilon}\right),\left(u^{\varepsilon}\right),\left(c^{\varepsilon}\right)$, not relabelled for convenience, and functions $p, u, c$ such that, as $\varepsilon \rightarrow 0$,

$$
\begin{aligned}
p^{\varepsilon} & \rightarrow p & & \text { strongly in } L^{2}\left(0, T ; H^{1}(\Omega)\right) \\
u^{\varepsilon} & \rightarrow u & & \text { strongly in }\left(L^{4}\left(\Omega_{T}\right)\right)^{d} \\
c^{\varepsilon} & \rightarrow c & & \text { weak- }{ }^{*} \text { in } L^{\infty}\left(\Omega_{T}\right) \\
c^{\varepsilon} u^{\varepsilon} & \rightarrow c u & & \text { strongly in }\left(L^{2}\left(\Omega_{T}\right)\right)^{d} .
\end{aligned}
$$

Moreover, the pair $(p, c)$ is a weak solution of the degenerate elliptic-parabolic system (1.8) in the sense of Definition 1.2.

This result is, in a way, an extension to the $d$-dimensional case of the one we obtained in [1] for the 1-dimensional case. We note (as in [1]) that the convergence of the sequence $\left(c^{\varepsilon}\right)$ is only weak-* in $L^{\infty}\left(\Omega_{T}\right)$, and Hypothesis (A5) is crucial in the proof of Theorem 1.3.

The next section is devoted to the proof of Theorem 1.3. Let us mention that uniqueness of the weak solution of the degenerate elliptic-parabolic system (1.7) is an open problem.

\section{Proof of Theorem $\mathbf{1 . 3}$}

The proof consists of two parts. In the first one we derive some estimates for $p^{\varepsilon}, u^{\varepsilon}, c^{\varepsilon}$ that are independent of $\varepsilon$. In the second part we pass to the limit, as $\varepsilon \rightarrow 0$, using compensated compactness techniques.

In the sequel, for convenience, the convergent extracted subsequences are not relabelled, i.e. they are denoted likewise the original sequences.

2.1 Some estimates. Let, for any $\varepsilon>0,\left(p^{\varepsilon}, c^{\varepsilon}\right)$ denote a corresponding weak solution to problem (1.7). We first establish the following estimates.

Lemma 2.1. The following assertions hold:

(i) The sequence $\left(p^{\varepsilon}\right)$ is bounded in $L^{\infty}\left(0, T ; H^{1}(\Omega)\right)$.

(ii) The sequences $\left(\sqrt{\varepsilon} \operatorname{grad} c^{\varepsilon}\right)$ and $\left(\left|u^{\varepsilon}\right|^{\frac{1}{2}} \operatorname{grad} c^{\varepsilon}\right)$ are bounded in the space $\left(L^{2}\left(\Omega_{T}\right)\right)^{d}$, and the sequences $\left(u^{\varepsilon} \cdot \operatorname{grad} c^{\varepsilon}\right)$ and $\left(D\left(u^{\varepsilon}\right) \operatorname{grad} c^{\varepsilon}\right.$. $\left.\operatorname{grad} c^{\varepsilon}\right)$ are bounded in the spaces $L^{2}\left(0, T ; L^{\frac{4}{3}}(\Omega)\right)$ and $L^{1}\left(\Omega_{T}\right)$, respectively.

(iii) The sequence $\left(p^{\varepsilon}\right)$ is bounded in $L^{2}\left(0, T ; W^{2, \frac{4}{3}}(\Omega)\right)$ and the sequence $\left(u^{\varepsilon}\right)$ is bounded in $\left(L^{2}\left(0, T ; W^{1, \frac{4}{3}}(\Omega)\right)\right)^{d}$.

Proof. Assertion (i). On multiplying the first equation in (1.7) by $p^{\varepsilon}$ and integrating over $\Omega$, we obtain

$$
\int_{\Omega} \frac{1}{\mu\left(c^{\varepsilon}\right)}\left|\operatorname{grad} p^{\varepsilon}\right|^{2} d x=\int_{\Omega}\left(q^{+}-q^{-}\right) p^{\varepsilon} d x .
$$


Using the inequality

$$
\int_{\Omega}\left|\left(q^{+}-q^{-}\right)\right| p^{\varepsilon} d x \leq \delta \int_{\Omega}\left|p^{\varepsilon}\right|^{2} d x+\frac{1}{4 \delta} \int_{\Omega}\left|q^{+}-q^{-}\right|^{2} d x
$$

for any $\delta>0$ and the Poincaré inequality we obtain

$$
\int_{\Omega} \frac{1}{\mu\left(c^{\varepsilon}\right)}\left|\operatorname{grad} p^{\varepsilon}\right|^{2} d x \leq \frac{1}{4 \delta} \int_{\Omega}\left|q^{+}-q^{-}\right|^{2} d x+C \delta \int_{\Omega}\left|\operatorname{grad} p^{\varepsilon}\right|^{2} d x
$$

where $C$ is a constant independent of $\varepsilon$. According hypothesis (A5) and choosing $\delta$ small enough, we infer that the sequence $\left(p^{\varepsilon}\right)$ is bounded in the space $L^{\infty}\left(0, T ; H^{1}(\Omega)\right)$, and therefore $\left(u^{\varepsilon}\right)$ is bounded in $\left(L^{\infty}\left(0, T ; L^{2}(\Omega)\right)\right)^{d}$.

Assertion (ii). We multiply the fourth equation of system (1.7) by $c^{\varepsilon}$ and integrate over $\Omega$. This gives

$$
\begin{aligned}
& \frac{1}{2} \frac{d}{d t} \int_{\Omega}\left|c^{\varepsilon}\right|^{2} d x+\int_{\Omega} D\left(u^{\varepsilon}\right) \operatorname{grad} c^{\varepsilon} \cdot \operatorname{grad} c^{\varepsilon} d x \\
& \quad=-\int_{\Omega}\left(u^{\varepsilon} \cdot \operatorname{grad} c^{\varepsilon}\right) c^{\varepsilon} d x+\int_{\Omega}\left(\hat{c}-c^{\varepsilon}\right) q^{+} c^{\varepsilon} d x .
\end{aligned}
$$

By Theorem 1.1 we have $0 \leq c^{\varepsilon}(x, t) \leq 1$, then

$$
\int_{\Omega}\left|\left(u^{\varepsilon} \cdot \operatorname{grad} c^{\varepsilon}\right) c^{\varepsilon}\right| d x \leq \delta \int_{\Omega}\left|u^{\varepsilon}\right|\left|\operatorname{grad} c^{\varepsilon}\right|^{2} d x+\frac{1}{4 \delta} \int_{\Omega}\left|u^{\varepsilon}\right| d x
$$

for any $\delta>0$. Using the first inequality of (1.5), we then have

$$
\begin{aligned}
& \frac{1}{2} \frac{d}{d t} \int_{\Omega}\left|c^{\varepsilon}\right|^{2} d x+\left(d_{t}-\delta\right) \int_{\Omega}\left|u^{\varepsilon}\right|\left|\operatorname{grad} c^{\varepsilon}\right|^{2} d x+\varepsilon \int_{\Omega}\left|\operatorname{grad} c^{\varepsilon}\right|^{2} d x \\
& \leq \frac{1}{4 \delta} \int_{\Omega}\left|u^{\varepsilon}\right| d x+\int_{\Omega}(\hat{c}+1)\left|q^{+}\right| d x .
\end{aligned}
$$

Integrating with respect to $t$ and choosing $\delta$ small enough, we obtain that the sequences $\left(\left|u^{\varepsilon}\right|^{\frac{1}{2}} \operatorname{grad} c^{\varepsilon}\right)$ and $\left(\sqrt{\varepsilon} \operatorname{grad} c^{\varepsilon}\right)$ are bounded in $\left(L^{2}\left(\Omega_{T}\right)\right)^{d}$. Then, writing

$$
\left|u^{\varepsilon} \cdot \operatorname{grad} c^{\varepsilon}\right| \leq\left|u^{\varepsilon}\right|\left|\operatorname{grad} c^{\varepsilon}\right|=\left|u^{\varepsilon}\right|^{\frac{1}{2}}\left|u^{\varepsilon}\right|^{\frac{1}{2}}\left|\operatorname{grad} c^{\varepsilon}\right|
$$

and using Hölder's inequality and the previous estimates, we find that the sequence $\left(u^{\varepsilon} \cdot \operatorname{grad} c^{\varepsilon}\right)$ is bounded in $L^{2}\left(0, T ; L^{\frac{4}{3}}(\Omega)\right)$. Then, by the last inequality of $(1.5)$, the sequence $\left(D\left(u^{\varepsilon}\right) \operatorname{grad} c^{\varepsilon} \cdot \operatorname{grad} c^{\varepsilon}\right)$ is bounded in $L^{1}\left(\Omega_{T}\right)$.

Assertion (iii). Writing the pressure equation in the form

$$
\begin{aligned}
-\Delta p^{\varepsilon} & =-\mu^{\prime}\left(c^{\varepsilon}\right) u^{\varepsilon} \cdot \operatorname{grad} c^{\varepsilon}+\mu\left(c^{\varepsilon}\right)\left(q^{+}-q^{-}\right) \\
\left.u^{\varepsilon} \cdot \nu\right|_{\Gamma_{T}} & =0 \\
\int_{\Omega} p^{\varepsilon}(x, t) d x & =0
\end{aligned}
$$


we deduce, following Grisvard [10], that

$\left(p^{\varepsilon}\right)$ is bounded in $L^{2}\left(0, T ; W^{2, \frac{4}{3}}(\Omega)\right)$

$\left(u^{\varepsilon}\right)$ is bounded in $\left(L^{2}\left(0, T ; W^{1, \frac{4}{3}}(\Omega)\right)\right)^{d}$.

Thus Lemma 2.1 is proved.

Now we investigate the limit behavior, as $\varepsilon \rightarrow 0$, of the weak solutions $\left(p^{\varepsilon}, c^{\varepsilon}\right)$ of problem (1.7). By Lemma 2.1, there are functions

$$
\begin{aligned}
& p \in L^{\infty}\left(0, T ; H^{1}(\Omega)\right) \\
& u \in\left(L^{\infty}\left(0, T ; L^{2}(\Omega)\right)\right)^{d} \\
& c \in L^{\infty}\left(\Omega_{T}\right)
\end{aligned}
$$

such that, for an appropriate subsequence,

$$
\begin{aligned}
& p^{\varepsilon} \rightarrow p \text { weak-* in } L^{\infty}\left(0, T ; H^{1}(\Omega)\right) \\
& u^{\varepsilon} \rightarrow u \text { weak-* in }\left(L^{\infty}\left(0, T ; L^{2}(\Omega)\right)\right)^{d} \\
& c^{\varepsilon} \rightarrow c \text { weak-* in } L^{\infty}\left(\Omega_{T}\right) .
\end{aligned}
$$

Note that, in virtue of Theorem $1.1,0 \leq c(x, t) \leq 1$ a.e. in $\Omega_{T}$.

The objective is to characterize the triplet $(p, u, c)$. First, we write the fourth equation of system (1.7) in the form

$$
\partial_{t} c^{\varepsilon}+\operatorname{div}\left(c^{\varepsilon} u^{\varepsilon}-D\left(u^{\varepsilon}\right) \operatorname{grad} c^{\varepsilon}\right)+c^{\varepsilon} q^{-}=\hat{c} q^{+} .
$$

After multiplication by a test function $\varphi$ in $C^{1}\left(\bar{\Omega}_{T}\right)$ with compact support in $\bar{\Omega} \times[0, T)$ and integration by parts, we obtain

$$
\begin{aligned}
\int_{\Omega_{T}} & \left\{c^{\varepsilon} \partial_{t} \varphi+\left(c^{\varepsilon} u^{\varepsilon}-D\left(u^{\varepsilon}\right) \operatorname{grad} c^{\varepsilon}\right) \cdot \operatorname{grad} \varphi-c^{\varepsilon} q^{-} \varphi\right\} d x d t \\
= & -\int_{\Omega_{T}} \hat{c} q^{+} \varphi d x d t-\int_{\Omega} c_{0}(x) \varphi(x, 0) d x
\end{aligned}
$$

It follows from the second inequality of (1.5) and Lemma 2.1/(ii) that the sequence $\left(D\left(u^{\varepsilon}\right) \operatorname{grad} c^{\varepsilon}\right)$ is bounded in $\left(L^{2}\left(0, T ; L^{\frac{4}{3}}(\Omega)\right)\right)^{d}$. Then letting $\varepsilon \rightarrow$ 0 in (2.1) yields

$$
\begin{gathered}
\int_{\Omega_{T}}\left\{c \partial_{t} \varphi+\overline{c u} \cdot \operatorname{grad} \varphi-\bar{D} \cdot \operatorname{grad} \varphi-c q^{-} \varphi\right\} d x d t \\
=-\int_{\Omega_{T}} \hat{c} q^{+} \varphi d x d t-\int_{\Omega} c_{0}(x) \varphi(x, 0) d x
\end{gathered}
$$


where, for extracted subsequences,

$$
\begin{array}{r}
c^{\varepsilon} u^{\varepsilon} \rightarrow \overline{c u} \text { weak-* in }\left(L^{\infty}\left(0, T ; L^{2}(\Omega)\right)\right)^{d} \\
D\left(u^{\varepsilon}\right) \operatorname{grad} c^{\varepsilon} \rightarrow \bar{D} \text { weakly in }\left(L^{2}\left(0, T ; L^{\frac{4}{3}}(\Omega)\right)\right)^{d} .
\end{array}
$$

We then have, in the sense of distributions in $\Omega_{T}$,

$$
\partial_{t} c+\operatorname{div}(\overline{c u})-\operatorname{div} \bar{D}+c q^{-}=\hat{c} q^{+}
$$

In what follows, we try to get more information on $\overline{c u}, u$ and $\bar{D}$.

2.2 Passing to the limit as $\varepsilon \rightarrow 0$. We begin by recalling the following compensated compactness result, derived from the characterization of compact sets of Aubin-Simon type as described in Lions [13], and due to Kazhikhov [11: Lemma 6].

Lemma 2.2. Let $V, W, V_{1}$ be Banach spaces such that

$$
\begin{array}{r}
\mathcal{D}(\Omega) \subset V \subset W \subset \mathcal{D}^{\prime}(\Omega) \\
V^{\prime} \subset V_{1}^{\prime} \subset \mathcal{D}^{\prime}(\Omega)
\end{array}
$$

with continuous embedding, the embedding $V \subset W$ being compact. Further, let $\left(\alpha^{\varepsilon}\right)$ and $\left(\beta^{\varepsilon}\right)$ be sequences such that, for some $1<p<\infty$,

$$
\begin{aligned}
& \left(\alpha^{\varepsilon}\right) \text { is bounded in } L^{p}(0, T ; V) \\
& \alpha^{\varepsilon} \rightarrow \alpha \text { weak in } L^{p}(0, T ; W) \\
& \left(\beta^{\varepsilon}\right) \text { is bounded in } L^{q}\left(0, T ; W^{\prime}\right) \\
& \beta^{\varepsilon} \rightarrow \beta \text { weak in } L^{q}\left(0, T ; W^{\prime}\right) \quad\left(q \geq \frac{p}{p-1}\right) \\
& \left(\partial_{t} \beta^{\varepsilon}\right) \text { is bounded in } L^{p_{1}}\left(0, T ; V_{1}^{\prime}\right) \quad\left(1 \leq p_{1} \leq \infty\right) \text {. }
\end{aligned}
$$

Then one can extract subsequences such that $\alpha^{\varepsilon} \beta^{\varepsilon} \rightarrow \alpha \beta$ in $\mathcal{D}^{\prime}\left(\Omega_{T}\right)$.

The aim is first to prove that $\overline{c u}=c u$.

Lemma 2.3. For extracted subsequences, $c^{\varepsilon} u^{\varepsilon} \rightarrow c u$ weak-* in the space $\left(L^{\infty}\left(0, T ; L^{2}(\Omega)\right)\right)^{d}$.

Proof. According to Lemma 2.1/(iii), it follows from the fourth equation of system (1.7) that $\left(\partial_{t} c^{\varepsilon}\right)$ is uniformly bounded in $L^{2}\left(0, T ; W^{-1, \frac{4}{3}}(\Omega)\right)$. Let

$$
V=W^{1, \frac{4}{3}}(\Omega), \quad W=L^{\frac{4}{3}}(\Omega), \quad V_{1}=W^{1,4}(\Omega) .
$$

Then $\mathcal{D}(\Omega) \subset V, W \subset \mathcal{D}^{\prime}(\Omega)$ and $V$ is compactly embedded into $W$. Moreover, $V_{1} \subset V$ so that the sequence $\left(\partial_{t} c^{\varepsilon}\right)$ is bounded in $L^{2}\left(0, T ;\left(W^{1,4}(\Omega)\right)^{\prime}\right)$. 
Since $0 \leq c^{\varepsilon}(x, t) \leq 1$ a.e. in $\Omega_{T}$, the sequence $\left(c^{\varepsilon}\right)$ is bounded in the space $L^{q}\left(0, T ; L^{4}(\Omega)\right)$ for any $q \geq 1$. Thus

$$
\begin{aligned}
& \left(u^{\varepsilon}\right) \text { is bounded in }\left(L^{2}(0, T ; V)\right)^{d} \\
& u^{\varepsilon} \rightarrow u \text { weak in }\left(L^{2}(0, T ; W)\right)^{d} \\
& \left(\partial_{t} c^{\varepsilon}\right) \text { is bounded in } L^{2}\left(0, T ; V^{\prime}\right) \\
& c^{\varepsilon} \rightarrow c \text { weak in } L^{2}\left(0, T ; W^{\prime}\right) .
\end{aligned}
$$

Then the proof follows from Lemma 2.2 and the fact that the sequence $\left(c^{\varepsilon} u^{\varepsilon}\right)$ is bounded in the space $\left(L^{\infty}\left(0, T ; L^{2}(\Omega)\right)\right)^{d}$.

We have the following characterization of the flux $u$ in terms of the pressure $p$ and the concentration $c$.

Lemma 2.4. The limit flux function $u$ is given by $u=-\frac{1}{\mu(c)} \operatorname{grad} p$.

Proof. We proceed as in [1]. The sequence $\left(\mu\left(c^{\varepsilon}\right)\right)$ is bounded in $L^{\infty}\left(\Omega_{T}\right)$. According to hypothesis (A5), there are $\bar{\mu}, \mu_{-1} \in L^{\infty}\left(\Omega_{T}\right)$ such that, for extracted subsequences, $\mu\left(c^{\varepsilon}\right) \rightarrow \bar{\mu}$ and $\frac{1}{\mu\left(c^{\varepsilon}\right)} \rightarrow \frac{1}{\mu-1}$ weak-* in $L^{\infty}\left(\Omega_{T}\right)$. We multiply the fourth equation of system $(1.7)$ by $\mu^{\prime}\left(c^{\varepsilon}\right)$. This writes in the form

$$
\begin{aligned}
\partial_{t} \mu\left(c^{\varepsilon}\right) & +\mu^{\prime}\left(c^{\varepsilon}\right) u^{\varepsilon} \cdot \operatorname{grad} c^{\varepsilon} \\
& +\mu^{\prime}\left(c^{\varepsilon}\right) c^{\varepsilon} q^{+}-\operatorname{div}\left(\mu^{\prime}\left(c^{\varepsilon}\right) D\left(u^{\varepsilon}\right) \operatorname{grad} c^{\varepsilon}\right) \\
= & -\mu^{\prime \prime}\left(c^{\varepsilon}\right) D\left(u^{\varepsilon}\right) \operatorname{grad} c^{\varepsilon} \cdot \operatorname{grad} c^{\varepsilon}+\mu^{\prime}\left(c^{\varepsilon}\right) \hat{c} q^{+} .
\end{aligned}
$$

In view of Lemma $2.1 /(\mathrm{ii})$, the right-hand side herein is bounded in $L^{1}\left(\Omega_{T}\right)$. Then the sequence $\left(\partial_{t} \mu\left(c^{\varepsilon}\right)\right)$ is bounded in $L^{2}\left(0, T ;\left(W^{1, \frac{4}{3}}(\Omega)\right)^{\prime}\right)$. Arguing as in Lemma 2.3 we obtain for a subsequence

$$
\mu\left(c^{\varepsilon}\right) u^{\varepsilon} \rightarrow \bar{\mu} u \quad \text { weak-* in }\left(L^{\infty}\left(0, T ; L^{2}(\Omega)\right)\right)^{d} \text {. }
$$

This gives $u=-\frac{\operatorname{grad} p}{\bar{\mu}}$.

We observe that $\frac{1}{\mu\left(c^{\varepsilon}\right)}$ satisfies

$$
\begin{aligned}
& \partial_{t}\left(\frac{1}{\mu\left(c^{\varepsilon}\right)}\right)-\frac{\mu^{\prime}\left(c^{\varepsilon}\right)}{\mu\left(c^{\varepsilon}\right)^{2}} u^{\varepsilon} \cdot \operatorname{grad} c^{\varepsilon} \\
& \quad-\frac{\mu^{\prime}\left(c^{\varepsilon}\right)}{\mu\left(c^{\varepsilon}\right)^{2}} c^{\varepsilon} q^{+}-\operatorname{div}\left(D\left(u^{\varepsilon}\right) \operatorname{grad}\left(\frac{1}{\mu\left(c^{\varepsilon}\right)}\right)\right) \\
& \quad=\frac{\left(\mu^{\prime \prime}\left(c^{\varepsilon}\right) \mu\left(c^{\varepsilon}\right)-2 \mu^{\prime}\left(c^{\varepsilon}\right)^{2}\right)}{\mu\left(c^{\varepsilon}\right)^{3}} D\left(u^{\varepsilon}\right) \operatorname{grad} c^{\varepsilon} \cdot \operatorname{grad} c^{\varepsilon}-\frac{\mu^{\prime}\left(c^{\varepsilon}\right)}{\mu\left(c^{\varepsilon}\right)^{2}} \hat{c} q^{+} .
\end{aligned}
$$


The sequence $\left(\frac{1}{\mu\left(c^{\varepsilon}\right)}\right)$ has the same properties as $\left(\mu\left(c^{\varepsilon}\right)\right)$, and the sequence $\left(\operatorname{grad} p^{\varepsilon}\right)$ has the same properties as $\left(u^{\varepsilon}\right)$. Then we can apply the same analysis. We obtain, for an appropriate subsequence,

$$
\frac{\operatorname{grad} p^{\varepsilon}}{\mu\left(c^{\varepsilon}\right)} \rightarrow \frac{\operatorname{grad} p}{\mu_{-1}}=-u \quad \text { weak-* in }\left(L^{\infty}\left(0, T ; L^{2}(\Omega)\right)\right)^{d} .
$$

We get $\bar{\mu} u=\mu_{-1} u$. Then, following Tartar [18], the convexity of the function $\mu$ gives $\bar{\mu} \geq \mu(c)$. By considering the function $\frac{1}{\mu}$ we get $\frac{1}{\mu_{-1}} \geq \frac{1}{\mu(c)}$. Therefore $\bar{\mu} \geq \mu(c) \geq \mu_{-1}$ and

$$
\bar{\mu} u=\mu(c) u=\mu_{-1} u=-\operatorname{grad} p .
$$

This ends the proof of Lemma 2.4.

Let us now state and prove the following result.

Lemma 2.5. Up to a subsequence, $\operatorname{grad} p^{\varepsilon} \rightarrow \operatorname{grad} p$ strongly in the space $\left(L^{2}\left(\Omega_{T}\right)\right)^{d}$.

Proof. According to Lemma 2.4, the weak limit $u$ satisfies

$$
\operatorname{div} u=q^{+}-q^{-}, \quad u=-\frac{1}{\mu(c)} \operatorname{grad} p, \quad u \cdot \nu=0 \text { on } \Gamma_{T} .
$$

We also have $\operatorname{div}\left(u^{\varepsilon}-u\right)=0$. After multiplication by $p^{\varepsilon}-p$ and integration over $\Omega_{T}$ we get

$$
\int_{\Omega_{T}} \operatorname{div}\left(u^{\varepsilon}-u\right)\left(p^{\varepsilon}-p\right) d x d t=-\int_{\Omega_{T}}\left(u^{\varepsilon}-u\right) \cdot \operatorname{grad}\left(p^{\varepsilon}-p\right) d x d t=0 .
$$

Then

$$
\begin{aligned}
\int_{\Omega_{T}} & \frac{1}{\mu\left(c^{\varepsilon}\right)}\left|\operatorname{grad}\left(p^{\varepsilon}-p\right)\right|^{2} d x d t \\
= & \int_{\Omega_{T}}\left(\frac{1}{\mu(c)}-\frac{1}{\mu\left(c^{\varepsilon}\right)}\right) \operatorname{grad} p \cdot \operatorname{grad}\left(p^{\varepsilon}-p\right) d x d t \\
= & \int_{\Omega_{T}}\left(u^{\varepsilon}+\frac{1}{\mu\left(c^{\varepsilon}\right)} \operatorname{grad} p\right) \cdot \operatorname{grad} p d x d t \\
& +\int_{\Omega_{T}} \frac{1}{\mu(c)} \operatorname{grad} p \cdot \operatorname{grad}\left(p^{\varepsilon}-p\right) d x d t .
\end{aligned}
$$

Sending $\varepsilon \rightarrow 0$, taking into account Lemma 2.4, the right-hand side herein converges to

$$
\int_{\Omega_{T}}\left(-\frac{1}{\mu(c)}+\frac{1}{\mu_{-1}}\right) \operatorname{grad} p \cdot \operatorname{grad} p d x d t
$$

and this limit is 0 , according to (2.5). To conclude, we note that the left-hand side of $(2.7)$ is bounded below by $\mu_{+}^{-1}\left\|\operatorname{grad}\left(p^{\varepsilon}-p\right)\right\|_{L^{2}\left(\Omega_{T}\right)}^{2}$. This completes the proof of Lemma 2.5. 
We can now establish results of strong convergence for the sequences $\left(u^{\varepsilon}\right)$ and $\left(c^{\varepsilon} u^{\varepsilon}\right)$.

Lemma 2.6. It holds that

$$
\begin{aligned}
u^{\varepsilon} & \rightarrow u & & \text { strongly in }\left(L^{2}\left(\Omega_{T}\right)\right)^{d} \\
c^{\varepsilon} u^{\varepsilon} & \rightarrow c u & & \text { strongly in }\left(L^{2}\left(\Omega_{T}\right)\right)^{d} \\
u^{\varepsilon} \cdot \operatorname{grad} c^{\varepsilon} & \rightarrow u \cdot \operatorname{grad} c & & \text { weak in } L^{2}\left(0, T ; L^{\frac{4}{3}}(\Omega)\right) .
\end{aligned}
$$

Proof. It follows from $(2.4)$ and $(2.5)$ that the sequence $\left(\mu\left(c^{\varepsilon}\right) u\right)$ converges weakly in $\left(L^{2}\left(\Omega_{T}\right)\right)^{d}$ to $\mu(c) u$. We have

$$
\mu\left(c^{\varepsilon}\right)-\mu(c)-\left(c^{\varepsilon}-c\right) \mu^{\prime}(c)=\frac{1}{2}\left(c^{\varepsilon}-c\right)^{2} \mu^{\prime \prime}\left(d^{\varepsilon}\right)
$$

with $d^{\varepsilon} \in(0,1)$ and, multiplying this relation by $u$,

$$
\mu\left(c^{\varepsilon}\right) u-\mu(c) u-\left(c^{\varepsilon}-c\right) \mu^{\prime}(c) u=\frac{1}{2}\left(c^{\varepsilon}-c\right)^{2} \mu^{\prime \prime}\left(d^{\varepsilon}\right) u .
$$

The left-hand side herein goes weakly to zero in $\left(L^{2}\left(\Omega_{T}\right)\right)^{d}$ as $\varepsilon \rightarrow 0$, then also the right-hand side. Hence

$$
\int_{\Omega_{T}} \mu^{\prime \prime}\left(d^{\varepsilon}\right)\left(c^{\varepsilon}-c\right)^{2} u \cdot u d x d t \rightarrow 0 .
$$

The strict convexity of $\mu$ implies that

$$
\int_{\Omega_{T}}\left(c^{\varepsilon}-c\right)^{2} u \cdot u d x d t \rightarrow 0 .
$$

This proves that the sequence $\left(c^{\varepsilon} u\right)$ converges strongly in $\left(L^{2}\left(\Omega_{T}\right)\right)^{d}$ to $c u$. It holds also that $\left(\mu\left(c^{\varepsilon}\right) u\right)$ converges strongly in $\left(L^{2}\left(\Omega_{T}\right)\right)^{d}$ to $\mu(c) u$. Then, writing

$$
\begin{aligned}
\mu\left(c^{\varepsilon}\right)\left|u^{\varepsilon}-u\right| & =\left|\mu\left(c^{\varepsilon}\right) u^{\varepsilon}-\mu\left(c^{\varepsilon}\right) u\right| \\
& \leq\left|\mu\left(c^{\varepsilon}\right) u^{\varepsilon}-\mu(c) u\right|+\left|\left(\mu(c)-\mu\left(c^{\varepsilon}\right)\right) u\right| \\
& =\left|\nabla p^{\varepsilon}-\nabla p\right|+\left|\left(\mu(c)-\mu\left(c^{\varepsilon}\right)\right) u\right|
\end{aligned}
$$

we deduce, using Lemma 2.5 and hypothesis (A5), that the sequence $\left(u^{\varepsilon}\right)$ converges strongly in $\left(L^{2}\left(\Omega_{T}\right)\right)^{d}$ to $u$. Then $(2.8)$ is proved.

Writing

$$
c^{\varepsilon} u^{\varepsilon}-c u=c^{\varepsilon}\left(u^{\varepsilon}-u\right)+\left(c^{\varepsilon}-c\right) u
$$

we conclude that the sequence $\left(c^{\varepsilon} u^{\varepsilon}\right)$ converges strongly in $\left(L^{2}\left(\Omega_{T}\right)\right)^{d}$ to $c u$, i.e. (2.9) is proved.

We note that the sequence $\left(u^{\varepsilon} \cdot \operatorname{grad} c^{\varepsilon}\right)$ is bounded in $L^{2}\left(0, T ; L^{\frac{4}{3}}(\Omega)\right)$ according to Lemma 2.1/(ii). Using the first equation of system (1.7) we have

$$
u^{\varepsilon} \cdot \operatorname{grad} c^{\varepsilon}=\operatorname{div}\left(c^{\varepsilon} u^{\varepsilon}\right)-c^{\varepsilon}\left(q^{+}-q^{-}\right) .
$$

Passing to the limit as $\varepsilon \rightarrow 0$, using (2.6) and (2.9) we deduce (2.10). This ends the proof of the lemma. 
The next part is concerned with the behavior of the dispersion term. For this purpose, let $\varphi \in C^{1}\left(\bar{\Omega}_{T}\right)$ with compact support in $\bar{\Omega} \times[0, T)$ and let

$$
\begin{aligned}
I^{\varepsilon}(\varphi) & =\int_{\Omega_{T}} D\left(u^{\varepsilon}\right) \operatorname{grad} c^{\varepsilon} \cdot \operatorname{grad} \varphi d x d t \\
& =\sqrt{\varepsilon} I_{1}^{\varepsilon}(\varphi)+\left(d_{l}-d_{t}\right) I_{2}^{\varepsilon}(\varphi)+d_{t} I_{3}^{\varepsilon}(\varphi)
\end{aligned}
$$

with

$$
\begin{aligned}
I_{1}^{\varepsilon}(\varphi) & =\sqrt{\varepsilon} \int_{\Omega_{T}} \operatorname{grad} c^{\varepsilon} \cdot \operatorname{grad} \varphi d x d t \\
I_{2}^{\varepsilon}(\varphi) & =\int_{\Omega_{T}} \frac{u^{\varepsilon}\left(u^{\varepsilon}\right)^{\top}}{\left|u^{\varepsilon}\right|} \operatorname{grad} c^{\varepsilon} \cdot \operatorname{grad} \varphi d x d t \\
I_{3}^{\varepsilon}(\varphi) & =\int_{\Omega_{T}}\left|u^{\varepsilon}\right| \operatorname{grad} c^{\varepsilon} \cdot \operatorname{grad} \varphi d x d t .
\end{aligned}
$$

The convergence of these integrals can be achieved as follows.

Applying the Cauchy-Schwarz inequality and using Lemma 2.1/(ii), we see that the sequence $\left(I_{1}^{\varepsilon}(\varphi)\right)$ is bounded and then $\lim _{\varepsilon \rightarrow 0} \sqrt{\varepsilon} I_{1}^{\varepsilon}(\varphi)=0$.

Further, we write $I_{2}^{\varepsilon}(\varphi)$ as

$$
I_{2}^{\varepsilon}(\varphi)=\int_{\Omega_{T}} \frac{u^{\varepsilon}}{\left|u^{\varepsilon}\right|^{\frac{1}{2}}} \cdot \operatorname{grad} c^{\varepsilon} \frac{u^{\varepsilon}}{\left|u^{\varepsilon}\right|^{\frac{1}{2}}} \cdot \operatorname{grad} \varphi d x d t .
$$

By (2.8), the sequence $\left(u^{\varepsilon}\right)$ converges strongly in $\left(L^{2}\left(\Omega_{T}\right)\right)^{d}$ to $u$, then there is an extracted subsequence such that $u^{\varepsilon} \rightarrow u$ a.e. in $\Omega_{T}$. From Lemma $2.1 /$ (iii) and the Sobolev embedding theorem, it follows that $\left(u^{\varepsilon}\right)$ is bounded in $\left(L^{2}\left(0, T ; L^{q}(\Omega)\right)\right)^{d}$ with $q=\frac{4 d}{3 d-4}$. Bearing in mind that $\left(u^{\varepsilon}\right)$ is also bounded in $\left(L^{\infty}\left(0, T ; L^{2}(\Omega)\right)\right)^{d}$, we deduce by interpolation that $\left(u^{\varepsilon}\right)$ is bounded in $\left(L^{r}\left(0, T ; L^{s} \Omega\right)\right)^{d}$ with $\frac{1}{r}=\frac{\alpha}{2}$ and $\frac{1}{s}=\frac{\alpha}{q}+\frac{1-\alpha}{r}$ where $0<\alpha<1$. We choose $\alpha$ so that $r=s$, i.e. $\alpha=\frac{2 d}{d+4}$, which gives $r=s=d+\frac{4}{d}$. Thus the sequence $\left(u^{\varepsilon}\right)$ is bounded in $\left(L^{r}\left(\Omega_{T}\right)\right)^{d}$ and we notice that $r>2$.

Consider now the continuous function $\Psi: \mathbb{R}^{d} \rightarrow \mathbb{R}^{d}$, defined by $\Psi(v)=\frac{v}{|v|^{\frac{1}{2}}}$ if $v \neq 0$ and $\Psi(v)=0$ otherwise. From the above considerations we deduce that $\Psi\left(u^{\varepsilon}\right) \rightarrow \Psi(u)$ a.e. in $\Omega_{T}$ and that the sequence $\left(\Psi\left(u^{\varepsilon}\right)\right)$ is bounded in $\left(L^{2 r}\left(\Omega_{T}\right)\right)^{d}$. Noticing that $2 r>4$ we deduce that, for an appropriate subsequence, $\Psi\left(u^{\varepsilon}\right) \rightarrow \Psi(u)$ strongly in $\left(L^{4}\left(\Omega_{T}\right)\right)^{d}$ which implies

$$
\Psi\left(u^{\varepsilon}\right) \cdot \operatorname{grad} \varphi \rightarrow \Psi(u) \cdot \operatorname{grad} \varphi \quad \text { strongly in } L^{4}\left(\Omega_{T}\right) .
$$

Similarly we have, for an appropriate subsequence,

$$
\left|u^{\varepsilon}\right|^{\frac{1}{2}} \rightarrow|u|^{\frac{1}{2}} \quad \text { strongly in } L^{4}\left(\Omega_{T}\right) .
$$


Let us denote $\xi^{\varepsilon}=\Psi\left(u^{\varepsilon}\right) \cdot \operatorname{grad} c^{\varepsilon}$. We know by Lemma $2.1 /$ (ii) that the sequence $\left(\xi^{\varepsilon}\right)$ is bounded in $L^{2}\left(\Omega_{T}\right)$. Then there is $\xi$ in $L^{2}\left(\Omega_{T}\right)$ such that, for an appropriate subsequence, $\xi^{\varepsilon} \rightarrow \xi$ in $L^{2}\left(\Omega_{T}\right)$ weak. We deduce with (2.12) that

$$
\lim _{\varepsilon \rightarrow 0} I_{2}^{\varepsilon}(\varphi)=\int_{\Omega_{T}} \xi \Psi(u) \cdot \operatorname{grad} \varphi d x d t
$$

From the relation $\xi^{\varepsilon}\left|u^{\varepsilon}\right|^{\frac{1}{2}}=u^{\varepsilon} \cdot \operatorname{grad} c^{\varepsilon}$, using (2.10) and (2.13), we deduce $\xi|u|^{\frac{1}{2}}=u \cdot \operatorname{grad} c$. This gives, for a.e. $(x, t) \in \Omega_{T}$ such that $u(x, t) \neq 0$,

$$
\xi(x, t)=\left(\frac{u}{|u|^{\frac{1}{2}}} \cdot \operatorname{grad} c\right)(x, t) .
$$

We notice that, for a.e. $(x, t) \in \Omega_{T}$ with $u(x, t)=0,(\xi \Psi(u) \cdot \operatorname{grad} \varphi)(x, t)=0$. Hence

$$
\xi \Psi(u) \cdot \operatorname{grad} \varphi=\frac{(u \cdot \operatorname{grad} c)(u \cdot \operatorname{grad} \varphi)}{|u|}
$$

and then

$$
\begin{aligned}
\lim _{\varepsilon \rightarrow 0} I_{2}^{\varepsilon}(\varphi) & =\int_{\Omega_{T}} \frac{(u \cdot \operatorname{grad} c)(u \cdot \operatorname{grad} \varphi)}{|u|} d x d t \\
& =\int_{\Omega_{T}} \frac{u u^{\top}}{|u|} \operatorname{grad} c \cdot \operatorname{grad} \varphi d x d t .
\end{aligned}
$$

To determine the limit of $I_{3}^{\varepsilon}(\varphi)$ as $\varepsilon \rightarrow 0$ we introduce the sequence given by $\zeta^{\varepsilon}=\left|u^{\varepsilon}\right|^{\frac{1}{2}} \operatorname{grad} c^{\varepsilon}$ so that

$$
I_{3}^{\varepsilon}(\varphi)=\int_{\Omega_{T}} \zeta^{\varepsilon} \cdot\left(\left|u^{\varepsilon}\right|^{\frac{1}{2}} \operatorname{grad} \varphi\right) d x d t .
$$

In view of Lemma $2.1 /(\mathrm{ii})$, there is $\zeta \in\left(L^{2}\left(\Omega_{T}\right)\right)^{d}$ such that, for an appropriate subsequence, $\zeta^{\varepsilon} \rightarrow \zeta$ weakly in $\left(L^{2}\left(\Omega_{T}\right)\right)^{d}$. We deduce from (2.13) that, for an appropriate subsequence, $\left|u^{\varepsilon}\right|^{\frac{1}{2}} \operatorname{grad} \varphi \rightarrow|u|^{\frac{1}{2}} \operatorname{grad} \varphi$ strongly in $\left(L^{4}\left(\Omega_{T}\right)\right)^{d}$ and then

$$
\lim _{\varepsilon \rightarrow 0} I_{3}^{\varepsilon}(\varphi)=\int_{\Omega_{T}} \zeta \cdot\left(|u|^{\frac{1}{2}} \operatorname{grad} \varphi\right) d x d t
$$

To characterize $\zeta$ we write

$$
\begin{aligned}
u_{i}^{\varepsilon} \zeta^{\varepsilon} & =u_{i}^{\varepsilon}\left|u^{\varepsilon}\right|^{\frac{1}{2}} \operatorname{grad} c^{\varepsilon} \\
& =\left|u^{\varepsilon}\right|^{\frac{1}{2}} \operatorname{grad}\left(c^{\varepsilon} u_{i}^{\varepsilon}\right)-c^{\varepsilon}\left|u^{\varepsilon}\right|^{\frac{1}{2}} \operatorname{grad} u_{i}^{\varepsilon}
\end{aligned}
$$

for any component $u_{i}^{\varepsilon}$ of $u^{\varepsilon} \quad(1 \leq i \leq d)$. Due to (2.9) and (2.13) we have, for an appropriate subsequence,

$$
\left|u^{\varepsilon}\right|^{\frac{1}{2}} \operatorname{grad}\left(c^{\varepsilon} u_{i}^{\varepsilon}\right) \rightarrow|u|^{\frac{1}{2}} \operatorname{grad}\left(c u_{i}\right) \quad \text { in }\left(\mathcal{D}^{\prime}\left(\Omega_{T}\right)\right)^{d}
$$


and note here that $|u|^{\frac{1}{2}} \operatorname{grad}\left(c u_{i}\right) \in\left(L^{1}\left(\Omega_{T}\right)\right)^{d}$.

We also have, for an appropriate subsequence,

$$
c^{\varepsilon}\left|u^{\varepsilon}\right|^{\frac{1}{2}} \rightarrow c|u|^{\frac{1}{2}} \quad \text { strongly in } L^{4}\left(\Omega_{T}\right) .
$$

Indeed, according to (2.9) and (2.13), one can extract subsequences such that

$$
c^{\varepsilon}\left|u^{\varepsilon}\right|^{\frac{1}{2}} \rightarrow c|u|^{\frac{1}{2}} \quad \text { weakly in } L^{4}\left(\Omega_{T}\right)
$$

and

$$
\int_{\Omega_{T}}\left|c^{\varepsilon}\right|^{2}\left|u^{\varepsilon}\right| d x d t=\int_{\Omega_{T}} c^{\varepsilon}\left|c^{\varepsilon} u^{\varepsilon}\right| d x d t \rightarrow \int_{\Omega_{T}} c^{2}|u| d x d t .
$$

Thus $c^{\varepsilon}\left|u^{\varepsilon}\right|^{\frac{1}{2}} \rightarrow c|u|^{\frac{1}{2}}$ strongly in $L^{2}\left(\Omega_{T}\right)$ and therefore, since the sequence $\left(c^{\varepsilon}\left|u^{\varepsilon}\right|^{\frac{1}{2}}\right)$ is bounded in $L^{2 r}\left(\Omega_{T}\right) \quad(2 r>4)$, one can extract subsequences such that (2.17) holds. This implies

$$
c^{\varepsilon}\left|u^{\varepsilon}\right|^{\frac{1}{2}} \operatorname{grad} u_{i}^{\varepsilon} \rightarrow c|u|^{\frac{1}{2}} \operatorname{grad} u_{i} \quad \text { in }\left(\mathcal{D}^{\prime}\left(\Omega_{T}\right)\right)^{d} .
$$

Note also that $c|u|^{\frac{1}{2}} \operatorname{grad} u_{i} \in\left(L^{1}\left(\Omega_{T}\right)\right)^{d}$. Passing to the limit in (2.15), using (2.16) and (2.18) we get

$$
u_{i} \zeta=u_{i}|u|^{\frac{1}{2}} \operatorname{grad} c
$$

for any component $(1 \leq i \leq d)$. Then, for a.e. $(x, t) \in \Omega_{T}$ such that $u(x, t) \neq$ 0 , which implies $u_{i}(x, t) \neq 0$ for some $1 \leq i \leq d$, (2.19) gives

$$
\zeta(x, t)=\left(|u|^{\frac{1}{2}} \operatorname{grad} c\right)(x, t) .
$$

We notice that, for a.e. $(x, t) \in \Omega_{T}$ with $u(x, t)=0,\left(\zeta \cdot|u|^{\frac{1}{2}} \operatorname{grad} \varphi\right)(x, t)=0$. Hence $\zeta \cdot|u|^{\frac{1}{2}} \operatorname{grad} \varphi=|u| \operatorname{grad} c \cdot \operatorname{grad} \varphi$ and then

$$
\lim _{\varepsilon \rightarrow 0} I_{3}^{\varepsilon}(\varphi)=\int_{\Omega_{T}}|u| \operatorname{grad} c \cdot \operatorname{grad} \varphi d x d t
$$

Collecting (2.11), (2.14) and (2.20) we conclude that

$$
\lim _{\varepsilon \rightarrow 0} I^{\varepsilon}(\varphi)=\int_{\Omega_{T}} D^{\circ}(u) \operatorname{grad} c \cdot \operatorname{grad} \varphi d x d t
$$

where $D^{\circ}(u)$ is given by (1.9) and the limit $\bar{D}$ in the second relation of $(2.2)$ is characterized by $\bar{D}=D^{\circ}(u) \operatorname{grad} c$. Therefore, $(p, u)$ satisfies the first three equations of system (1.8) and equation (2.3) can be written also in form of the fourth equation of system (1.8). The proof of Theorem 1.3 is now complete. 


\section{References}

[1] Amirat, Y. and A. Ziani: Global weak solutions for a degenerate parabolic system modelling a one-dimensional compressible miscible flow in porous media. Boll. U.M.I. (to appear).

[2] Anderson, M. P.: Movement of contaminants in groundwater: groundwater transport - advection and dispersion. In: Groundwater Contamination, Studies in Geophysics, National Research Council. Washington: Nat. Acad. Press 1984, pp. $37-45$.

[3] Bear, J.: Dynamics of Fluids in Porous Media. New York: American Elsevier 1972 .

[4] Chavent, G. and J. Jaffré: Mathematical Methods and Finite Elements for Reservoir Simulation (Studies in Mathematics and its Applications: Vol. 17). Amsterdam: North-Holland Publ. Comp. 1986.

[5] Chen, Z. and R. E. Ewing: Mathematical analysis for reservoir models. SIAM J. Math. Anal. 30 (1999), 431 - 453.

[6] Coutinho, A. L. G. A. and J. L. D. Alves: Finite element simulation of nonlinear viscous fingering in miscible displacements with anisotropic dispersion and nonmonotonic viscosity profiles. Comput. Mech. 23 (1999), 108 - 116.

[7] Douglas, J.: Numerical Methods for the Flow of miscible Fluids in Porous Media (Computational Methods in Coupled Systems, eds.: R. W. Lewis et al.). New York: John Wiley 1984.

[8] Fabrie, P. and M. Langlais: Mathematical analysis of miscible displacement in porous media. SIAM J. Math. Anal. 23 (1992), 1375 - 1392.

[9] Feng, X.: On existence and uniqueness results for a coupled system modeling miscible displacement in porous media. J. Math. Anal. Appl. 194 (1995), 883 -910 .

[10] Grisvard, P.: Elliptic Problems in Nonsmooth Domains (Monographs and Studies in Mathematics: Vol. 24). London: Pitman 1985.

[11] Kazhikhov, A. V.: Recent developments in the global theory of two-dimensional compressible Navier-Stokes equations. Keio University (Yokohama, Japan): Sem. Math. Sciences 25 (1998).

[12] Koval, E. J.: A method for predicting the performance of unstable miscible displacements in heterogeneous media. Soc. Pet. Eng. J. (June 1963), 145 154.

[13] Lions, J. L.: Quelques méthodes de résolution des problèmes aux limites non linéaires. Paris: Dunod-Gauthier-Villars 1969.

[14] Mikelić, A.: Mathematical theory of stationary miscible filtration. J. Diff. Equ. 90 (1991), 186 - 202.

[15] Pearson, J. R. A. and P. M. J. Tardy: Models for flow of non-Newtonian and complex fluids through porous media. J. Non-Newtonian Fluid Mech. 102 (2002), $447-473$. 
[16] Scheidegger, A.E.: The Physics of Flow through Porous Media. Toronto: Univ. Toronto Press 1974.

[17] Shubin, G. R. and J. B. Bell: An analysis of the grid orientation effect in numerical simulation of miscible displacement. Comp. Meth. in Appl. Mech. \& Eng. 47 (1984), $47-71$.

[18] Tartar, L.: Compensated Compactness and Applications to P.D.E. Res. Notes in Math. 4 (39) (1979), 136 - 212.

[19] Young, L. C.: A study of spatial approximations for simulating fluid displacements in petroleum reservoirs. Comp. Methods in Appl. Mech. \& Eng. 47 (1984), $3-46$.

Received 14.07.2003; in revised form 4.11.2003 